\title{
Estigma na doença mental: estudo observacional
}

Ana Rita Fernandes Oliveira,* Sónia Meira Azevedo**

\section{RESUMO}

Objetivos: Verificar se existe estigma relativamente à doença mental na população em estudo e sua associação com características sociodemográficas ou contacto com doença mental.

Tipo de estudo: Analítico, transversal.

Local: Unidade de Saúde Familiar Santiago (Centro de Saúde Arnaldo Sampaio) e Centro de Saúde da Marinha Grande.

População: Utentes com idade igual ou superior a 18 anos inscritos nas duas unidades de saúde.

Métodos: Foi selecionada uma amostra não aleatória de conveniência e foram avaliadas variáveis sociodemográficas, antecedentes pessoais e familiares de doença mental e aplicado o questionário AQ-9 de Corrigan para medição do estigma. Análise univariável e multivariável, n.s. $=0,05$.

Resultados: Amostra de 206 utentes com média de 45 anos. Questões sobre ajuda, coerção para tratamento e pena foram aquelas que obtiveram maior classificação no questionário. Utentes com filhos e casados apresentaram maior concordância com coerção ( $t$-student $p=0,008$; ANOVA $p=0,018$ ). Baixa escolaridade associou-se a maior score na segregação dos doentes mentais (ANOVA $p<0,001$ ). Não se verificou associação entre o estigma e a presença de antecedentes pessoais ou familiares de doença mental.

Conclusões: Verificou-se existência de estigma na população estudada. O estado civil, a existência de filhos e a escolaridade apresentaram associação com o estigma. Não se encontrou associação com as restantes questões do AQ-9.

Palavras-chave: Estigma; Doença Mental.

\section{INTRODUÇÃO}

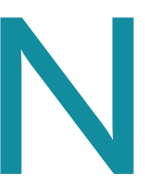
ão existe uma definição de doença mental que seja consensual do ponto de vista clínico, filosófico e científico. Quando os sintomas da doença são sentimentos, pensamentos e comportamentos, tendo em conta que as conexões entre o cérebro, a mente e o comportamento permanecem ainda em fase de exploração, mais difícil se torna o processo de elaboração de definições. ${ }^{1}$

Em 2001, a Organização Mundial de Saúde (OMS) definiu a perturbação mental como uma adulteração do pensamento e das emoções produzida por desadequação ou deterioração do funcionamento psicossocial em dependência de fatores biológicos, psicológicos e sociais. ${ }^{1}$

Estigma define-se como um atributo, visível ou não, que denuncia uma imperfeição no indivíduo, que o desqualifica de uma aceitação comunitária plena, resultando em atitudes discriminatórias, ou seja, o estigma pode

\footnotetext{
*Interna Formação Específica Medicina Geral e Familiar, USF Santiago, Leiria, Portugal **Interna Formação Específica Medicina Geral e Familiar, CS Marinha Grande, Marinha Grande, Portugal
}

ser definido como uma desaprovação social severa devido a características presumidas ou verídicas, crenças ou comportamentos, que não estão de acordo com as normas económicas, políticas, culturais ou sociais. ${ }^{2}$

O estigma da doença mental permanece um assunto complexo na sociedade, culminando em efeitos nefastos nos doentes mentais, retardando ou impedindo a procura de cuidados de saúde, a instituição ou manutenção de tratamento e a sua recuperação. ${ }^{3-6}$

Por parte da pessoa com doença mental, experienciar o estigma e a discriminação pode aumentar os níveis de stresse angústia e conduzir a uma diminuição do seu funcionamento psicossocial. ${ }^{2,5}$ Podem surgir sentimentos de raiva, tristeza e desencorajamento e, consequentemente, depressão, ansiedade e baixa autoestima, aspetos que irão contribuir para a diminuição do investimento por parte do próprio doente no seu processo de recuperação. ${ }^{1,3,7} \mathrm{O}$ conceito de recuperação é multidimensional e consiste num processo que possibilita ao indivíduo com doença mental maximizar a esperança relativamente ao futuro, envolver-se em atividades significativas e desen- 
volver a autodeterminação integrado na sociedade sem sofrer efeitos de estigma e discriminação..$^{1-2,6}$

Segundo a Associação Mundial de Psiquiatria, o estigma cria um ciclo vicioso de exclusão social e discriminação, constituindo uma enorme barreira para a qualidade de vida das pessoas com doença mental e dos seus familiares, por vezes até mais importante do que a própria doença. Procura-se que cada vez mais as pessoas com doença mental sejam tratadas em estruturas comunitárias; contudo, o peso de uma opinião pública negativa pode ter muito mais impacto no doente e no seu sistema familiar. Consequências do estigma como desemprego, autoestima diminuída, falta de habitação própria e fraco suporte social constituem grandes obstáculos ao processo de recuperação. Além disso, o estigma e expectativas de estigma, nomeadamente autoestigma, podem produzir desagregação do sistema familiar e reduzir o fluxo normal das interações sociais pelo desejo de manter em segredo a condição de doente mental. ${ }^{1}$

A deteção e abordagem do estigma na população utilizadora dos cuidados de saúde primários torna-se importante, visto que o médico de família encontra-se em posição privilegiada para educar e informar os utentes e as suas famílias, de modo a minimizar os efeitos do estigma na população. Para além disso, a importância deste estudo prende-se com a inexistência de dados na população da área de influência das autoras.

Este trabalho tem como objetivos: i) estimar a prevalência de estigma relativamente à doença mental na população em estudo; ii) verificar se existe associação do estigma com características sociodemográficas (idade, género, escolaridade, estado civil, existência de filhos) e iii) verificar se o contacto com doença mental (antecedentes pessoais ou familiares) influencia a existência de estigma.

\section{MÉTODOS}

Estudo analítico, transversal, que teve por base uma população de utentes inscritos em duas unidades de saúde do distrito de Leiria: USF Santiago (Leiria) e Centro de Saúde da Marinha Grande.

Foi selecionada uma amostra não aleatória, de conveniência, constituída por todos os utentes com idade igual ou superior a 18 anos, que se dirigiram ao Centro de Saúde da Marinha Grande e à USF Santiago durante o mês de abril de 2013 e que acederam a participar no estudo. Foram definidos como critérios de exclusão a recusa na participação no estudo e o preenchimento incompleto ou incorreto dos questionários.

A recolha de dados teve por base um questionário de autopreenchimento constituído por três grupos: a) grupo 1 com questões relacionadas com aspetos pessoais e sociodemográficos (idade, género, escolaridade, estado civil, existência de filhos); b) grupo 2 constituído por questões relacionadas com o contacto com a doença mental (história pessoal ou contacto familiar); c) grupo 3 constituído pelo Questionário de Atribuição - AQ-9 criado por Corrigan (versão preliminar portuguesa, revista e abreviada do Questionário de Atribuição-AQ-27 de Corrigan ${ }^{8}$ que pretende medir globalmente o estigma através de 9 fatores: "responsabilidade”, "pena”, "irritação”, "perigosidade”, "medo", “ajuda”, “coerção”, "segregação” e "evitamento", cotados pela soma dos itens segundo uma grelha específica. Este questionário, usado no domínio da investigação, pretende avaliar nove estereótipos acerca da doença mental, sendo constituído por uma descrição de um caso de uma pessoa com esquizofrenia, seguindo-se 9 afirmações ou questões para as quais existe uma escala de Likert de 1 a 9, em que o 1 corresponde maioritariamente à resposta "não ou nada" e o 9 a "muito ou completamente". O resultado produz um score representativo de cada um dos estereótipos, sendo que o estigma é diretamente proporcional ao valor do score. Admite-se, assim, que um resultado superior a 1 implica existência de estigma.

O questionário (figura 1) e respetivo consentimento informado foram distribuídos pelo administrativo aquando da efetivação da inscrição da consulta ou pelas próprias médicas durante a mesma. Após o preenchimento dos questionários e respetivos consentimentos informados foi solicitado aos doentes que procedessem à sua colocação, separadamente, em urna colocada para o efeito no balcão da sala de espera.

Previamente à recolha dos dados, foi aplicado o questionário em formato pré-teste, com algumas questões abertas direcionadas para a deteção de problemas na aplicabilidade do mesmo. Este pré-teste foi aplicado a uma amostra de 10 pessoas com idade igual ou superior a 18 anos, pertencentes a unidades de saúde diferentes das incluídas no estudo.

O protocolo do estudo foi submetido a avaliação pela Comissão de Ética da ARS do Centro, tendo obtido um parecer positivo e o estudo foi igualmente avaliado e 


\section{QUESTIONÁRIO}

Com este questionário pretendemos realizar um estudo sobre estigma em relação à doença mental. Gostaríamos que colaborasse no preenchimento deste questionário confidencial e anónimo. Responda com sinceridade.

Assinale a sua resposta com um $\mathbf{X}$.

Género:
$\square$ Masculino
Feminino
Idade: anos

1. Estado Civil ?

$\square$ Solteiro

Casado/União de facto

$\square$ Viúvo

Divorciado

2. Tem filhos?

$\square$ Não

Sim

3. Qual a sua escolaridade?
Analfabeto
4. ${ }^{\circ}$ ano
6. ano
9. ${ }^{\circ}$ ano
$12 .^{\circ}$ ano
Ensino superior

4. Sofre ou já sofreu de alguma doença mental (Depressão, Esquizofrenia, Doença Bipolar, Ansiedade, outra)?

$\square$ Não $\quad \square \operatorname{Sim}$

5. Teve algum contacto com alguém na sua família que sofresse de doença mental?

Não

$\square$ Sim

Se respondeu afirmativamente a esta questão:

Qual o grau de parentesco?
$\square$ Mãe
$\square$ Irmãos
$\square$ Tios
Outro. Qual?
Pai
Avós
Primos

6. Por favor leia a história do José:

O José é um homem com 30 anos de idade, solteiro e com Esquizofrenia. Às vezes ouve vozes e fica perturbado. Ele vive sozinho num apartamento e trabalha como estafeta num grande escritório de advogados. Já foi internado seis vezes devido à sua doença.

Agora responda a cada uma das seguintes questões sobre o José. Assinale com um $\mathbf{X}$ o número que melhor corresponde à sua resposta. (Numa escala de 1 a 9, em que 1 corresponde a "não ou nada" e 9 corresponde a "muito ou completamente").

\begin{tabular}{|c|c|c|c|c|c|c|c|c|c|}
\hline \multirow[t]{3}{*}{ Pergunta/Afirmação } & \multicolumn{9}{|c|}{ Resposta } \\
\hline & \multicolumn{5}{|c|}{$\begin{array}{l}\text { Não ou } \\
\text { nada }\end{array}$} & \multicolumn{4}{|c|}{$\begin{array}{r}\text { Muito ou } \\
\text { completamente }\end{array}$} \\
\hline & 1 & 2 & 3 & 4 & 5 & 6 & 7 & 8 & 9 \\
\hline Eu sentiria pena do José. & & & & & & & & & \\
\hline Quão perigoso acha que o José é? & & & & & & & & & \\
\hline Quão assustado do José se iria sentir? & & & & & & & & & \\
\hline Acho que o José é culpado da sua condição presente. & & & & & & & & & \\
\hline $\begin{array}{l}\text { Acho que seria melhor para a comunidade do José } \\
\text { se ele fosse internado num Hospital Psiquiátrico. }\end{array}$ & & & & & & & & & \\
\hline Quão zangado se sentiria com o José? & & & & & & & & & \\
\hline Qual a probabilidade com que iria ajudar o José? & & & & & & & & & \\
\hline Eu iria tentar manter-me afastado do José. & & & & & & & & & \\
\hline $\begin{array}{l}\text { Acha que o José devia ser forçado a fazer tratamento } \\
\text { médico mesmo contra a sua vontade? }\end{array}$ & & & & & & & & & \\
\hline
\end{tabular}

Figura 1. Questionário aplicado no estudo. 
aceite pela Direção das unidades de saúde em causa (USF Santiago e Centro de Saúde da Marinha Grande)

Os dados obtidos foram codificados e introduzidos numa base de dados, tendo sido depois tratados com a aplicação informática de estatística SPSS 18.0®. Foi efetuada análise estatística descritiva univariável e multivariável com a aplicação dos testes: $t$ de Student, MannWhitney, ANOVA e correlação de Pearson ou respetivos não paramétricos para um nível de significância de 0,05.

\section{RESULTADOS}

Entre os utentes inscritos em ambas as unidades de saúde obtiveram-se 210 respostas ao questionário. Foram excluídos 4 por preenchimento inadequado, pelo que se consideraram 206 questionários para estudo.

A maioria dos respondentes era do sexo feminino $(68,4 \% ; \mathrm{n}=141)$. As idades distribuíram-se entre um mínimo de 18 e um máximo de 85 anos, com média de $45,0 \pm 15,6$ anos. A maior parte dos indivíduos eram casados ou viviam em união de facto $(62,1 \% ; n=128)$ e tinham filhos $(75,7 \% ; n=156)$. Apenas 20,9\% $(n=43)$ tinham instrução superior, sendo que mais de metade $(51,9 \% ; \mathrm{n}=107)$ apresentou escolaridade igual ou inferior ao $3 .^{\circ}$ ciclo do ensino básico.

Relativamente ao contacto com doença mental, $37,9 \%$ $(n=78)$ dos utentes tinham antecedentes pessoais de doença enquanto mais de um terço $(36,9 \% ; n=76)$ tinha contacto com familiares com patologia deste foro, sendo na maioria $(19,4 \% ; n=40)$ familiares de primeiro grau.

Quando confrontados com o relato de um doente com esquizofrenia, as respostas às perguntas do questionário AQ-9, segundo uma escala de likert de 1 a 9 pontos, revelaram posições na sua maioria medianas, como se pode observar na Quadro I. Nas questões que avaliam a culpa, raiva e evitação do doente, a média das respostas revelou-se inferior a 3. As restantes afirmações despoletaram respostas em média entre 4,2 e 6,4. A moda observada em todas as respostas foi sempre um dos extremos da escala (pena, ajuda e coerção obtiveram moda de 9 , sendo que nas restantes alíneas se verificou moda de 1). (Figura 2)

Não se encontram diferenças estatisticamente significativas nas respostas ao AQ-9 consoante o género, idade ou história de antecedentes pessoais ou familiares de doença mental (Quadro II e IV). Utentes com filhos e casados apresentaram uma maior probabilidade de coerção para o tratamento do doente mental

\begin{tabular}{|lc|}
\hline QUADRO I. Médias obtidas para as 9 questões do AQ-9. \\
& Média \\
Pena & 5,54 \\
Perigosidade & 4,16 \\
Medo & 4,56 \\
Culpa & 1,51 \\
Segregação & 4,16 \\
Raiva & 2,86 \\
Ajuda & 6,42 \\
Evitação & 2,20 \\
Coerção & 6,00 \\
\hline
\end{tabular}

( $t$-student $p=0,008 ;$ ANOVA $p=0,018$ ) (Quadro III). Verificou-se ainda a existência de associação estatisticamente significativa entre a baixa escolaridade e um maior scorena questão relativa à segregação dos doentes mentais (ANOVA $p<0,001)$. (Quadro II)

\section{DISCUSSÃO}

Trinta e sete por cento dos utentes inquiridos referiram a existência de antecedentes prévios ou atuais de doença mental. Esta prevalência revela-se um pouco superior aos dados encontrados para a população portuguesa. Estima-se que a prevalência de perturbações psiquiátricas na população geral ronde os $30 \%$, sendo aproximadamente de $12 \%$ a de perturbações psiquiátricas graves, embora não existam dados de morbilidade psiquiátrica de abrangência nacional que permitam uma melhor caracterização do País. ${ }^{9}$

Esta elevada prevalência poderá, contudo, dever-se ao perfil dos hiperfrequentadores (género feminino, a idade avançada, a baixa escolaridade e a presença de patologia psiquiátrica) ${ }^{10}$ ou a uma errada perceção do conceito de doença mental, podendo os inquiridos ter englobado na resposta alguns sintomas psiquiátricos sem critérios patológicos, assim como patologias do foro neurológico, tendo em conta que não foi pedido ao doente que especificasse a perturbação nem foi dado ao doente uma definição de doença mental ou uma lista de diagnósticos passíveis de serem considerados na questão.

Relativamente às respostas obtidas no AQ-9 constatou-se, à semelhança do encontrado num estudo de 


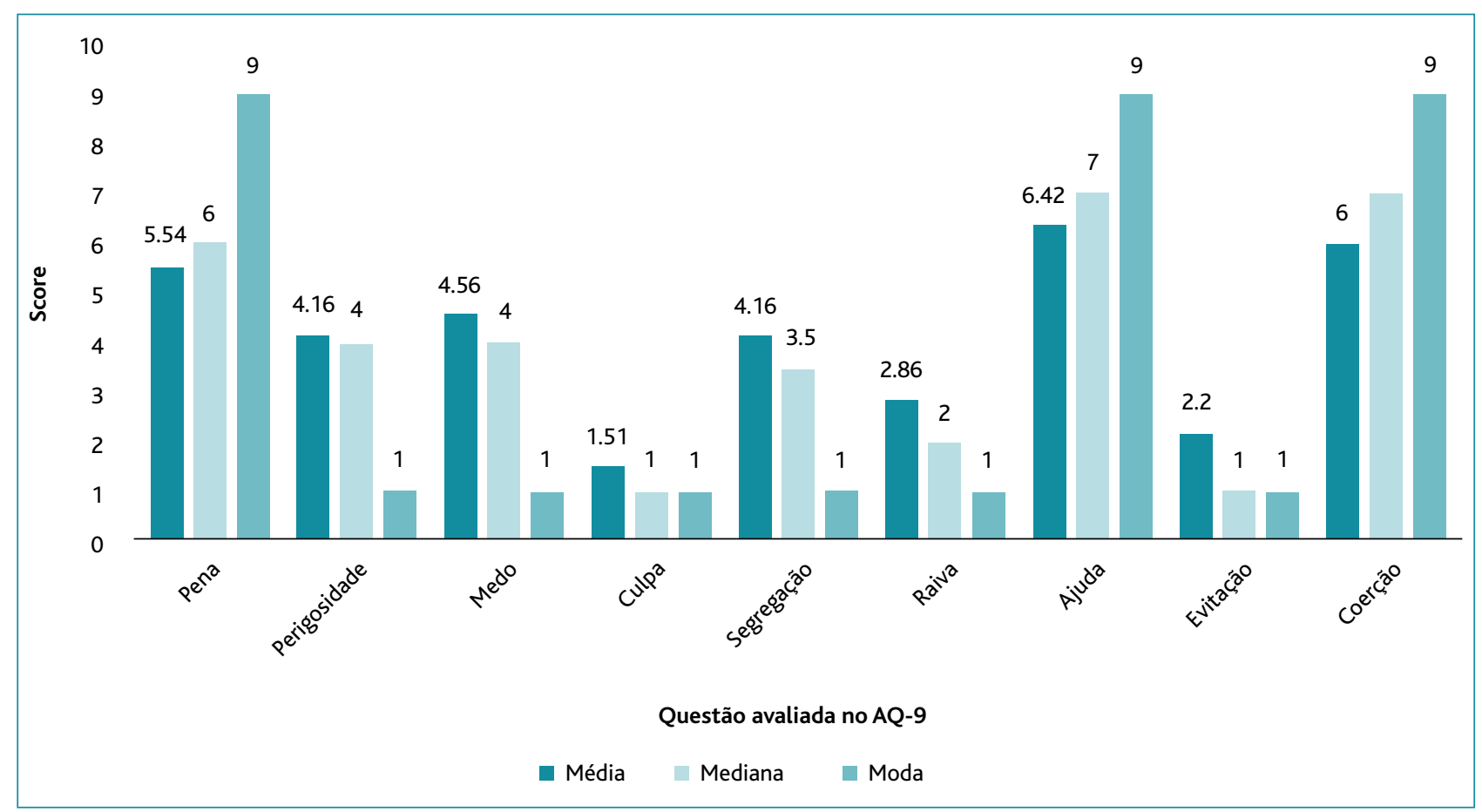

Figura 2. Discriminação da média, moda e mediana nas respostas do AQ-9 na amostra em estudo.

Barbosa $^{1}$ que teve por base a aplicação do AQ-27 (versão completa do AQ-9), que os parâmetros que obtiveram um maior grau de concordância foram os relativos à pena, ajuda e coerção para o tratamento (com uma média de 5,5 no estudo de Barbosa e médias ainda superiores no nosso estudo). Considerando que, quanto mais elevado o valor do score maior o estigma existente, pode admitir-se que o estigma está presente na amostra em estudo, uma vez que a maioria dos parâmetros avaliados no AQ-9 apresenta média superior a 4. Por outro lado, os que apresentaram médias mais baixas (inferiores a 3), ou seja, maior grau de discordância, foram a culpa, a raiva e a evitação.

No entanto, há que salientar o facto das modas se terem situado nos extremos. Esta concentração de resposta nos extremos poderá significar uma dificuldade de compreensão do questionário ou alertar-nos para o facto dos inquiridos terem dado a resposta que consideravam moralmente aceite.

Ainda que se tenha verificado uma maior probabilidade de coerção para o tratamento do doente mental nos utentes com filhos e casados e uma associação estatisticamente significativa entre a baixa escolaridade e um maior score na questão relativa à segregação dos doentes mentais $(A N O V A p<0,001)$ não se pode excluir que estes resultados se devam a um erro aleatório tipo 1.

Após a análise realizada, com base nesta amostra, admite-se que o género, a idade e o contacto com a doença mental não apresentem associação com o estigma. Contudo, dados da literatura apontam para o facto de, tanto a educação quanto o contacto com a doença mental, terem um impacto positivo na redução do estigma para com o doente mental. ${ }^{11}$

Um estudo polaco revelou que "a profissão, a frequência de contacto com o doente mental, o grau de literacia sobre a doença mental, a experiência pessoal, o nível de escolaridade, fatores culturais, o género e a idade são os fatores mais influentes/determinantes para a perceção do doente psiquiátrico, sendo que a literacia sobre a doença mental é o fator modificável que mais facilmente poderá ser passível de intervenção". ${ }^{12} \mathrm{Se}-$ gundo Corrigan et $\mathrm{al}^{13}$ as mulheres têm uma menor tendência a estigmatizar, assim como doentes com maior nível de escolaridade. ${ }^{13}$

Em jeito de conclusão, admite-se que existe estigma na população estudada e que o estado civil, a existên- 


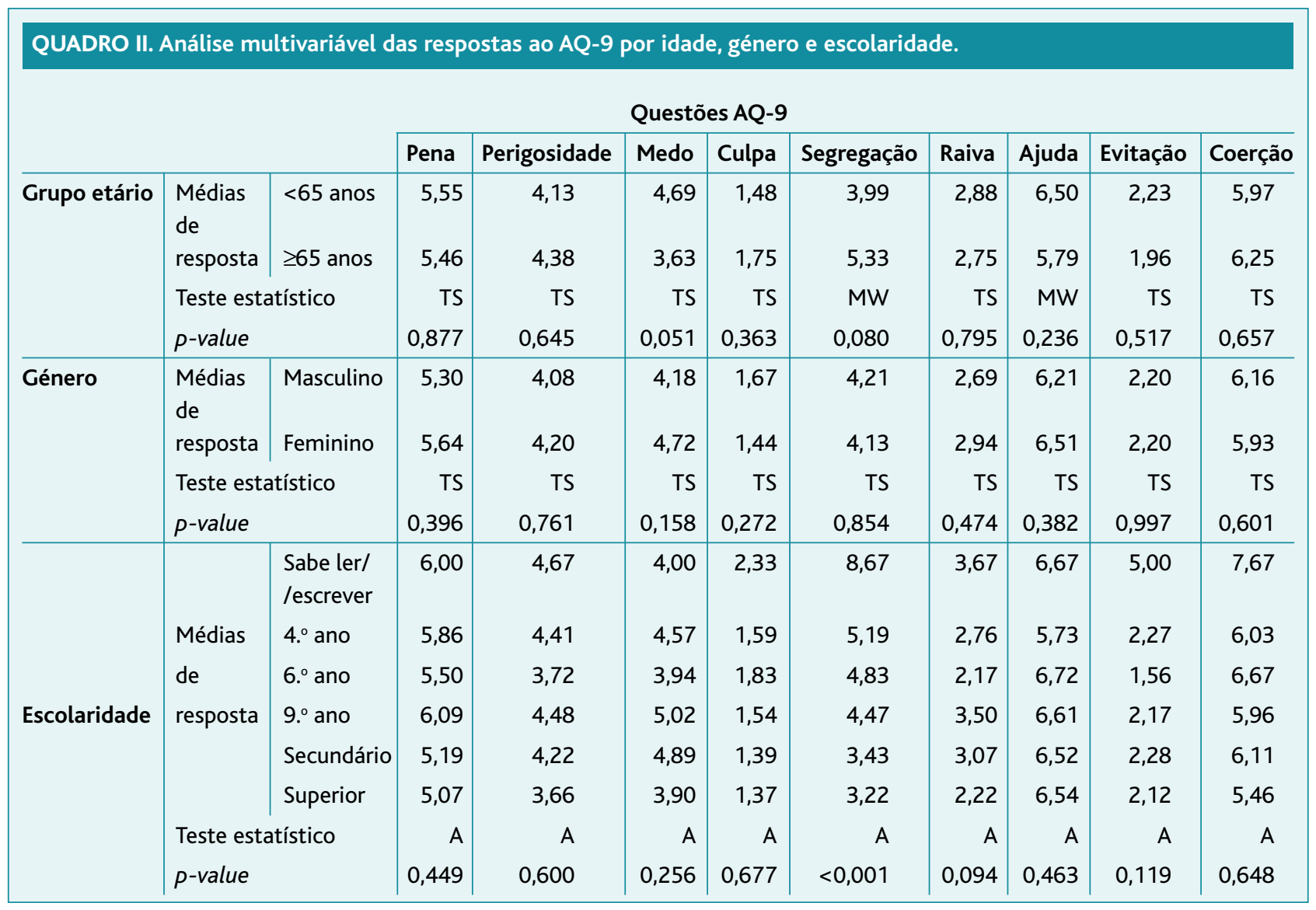

Legenda: TS: T-Student; MW; Mann-Whitney; A: ANOVA.

cia de filhos e a escolaridade influenciam esse mesmo estigma. Não se verificou associação entre a existência de estigma e as restantes variáveis estudadas.

Uma das principais limitações deste estudo prendese com o facto de se ter utilizado um questionário ainda em processo de validação para a população portuguesa; no entanto, tentou minimizar-se o erro através da aplicação de um pré-teste. Há que ter em conta que, ainda que o questionário tenha sido de autopreenchimento e anónimo, a pressão de responder o moralmente correto poderá ter condicionado as respostas. Não é ainda possível excluir a existência de um viés de seleção, tendo em conta que se utilizou uma amostra de conveniência não aleatorizada; contudo tentou minimizar-se este viés através do uso de uma amostra de tamanho alargado.

De futuro seria interessante aprofundar o estudo através da utilização de outra ferramenta de medição mais objetiva e da inclusão de uma questão que especifique as patologias psiquiátricas nos doentes que afirmem padecer de doença mental. Seria também útil inquirir o doente relativamente a situações de descriminação, vivenciadas pelos mesmos, decorrentes da sua patologia.

Está ainda em vista a realização de um estudo em parceria com o Serviço de Psiquiatria do Centro Hospitalar de Leiria, longitudinal, com uma amostra mais alargada, com vista a avaliar estes parâmetros numa amostra hospitalar, assim como a avaliar o estigma no próprio doente do foro mental no seio familiar e nos profissionais de saúde (através da aplicação, respetivamente, dos questionários The self stigma of mental illness scale (SSMIS), Family Questionnaire e AQ-9 modificado). ${ }^{8}$

Este trabalho de investigação poderá ser uma maisvalia porque é um estudo inovador e permite detetar fatores passíveis de serem alvo de medidas que visem a redução das atitudes discriminatórias e que facilitem a 


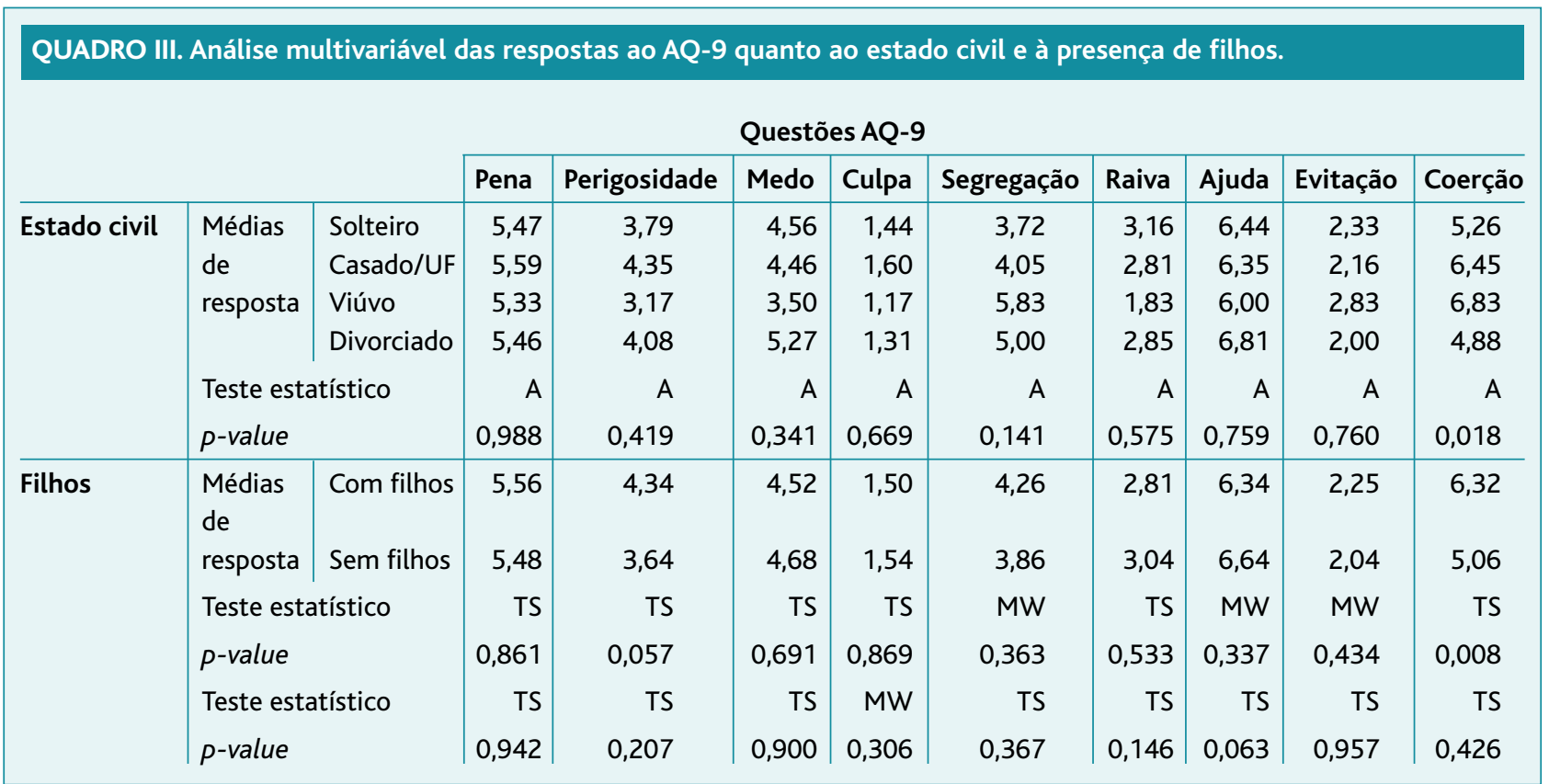

Legenda:TS:T-Student; MW; Mann-Whitney; A: ANOVA.

QUADRO IV. Análise multivariável das respostas ao AQ-9 consoante contacto com doença mental.

\begin{tabular}{|c|c|c|c|c|c|c|c|c|c|c|c|}
\hline & \multicolumn{9}{|c|}{ Questões AQ-9 } \\
\hline & & & Pena & Perigosidade & Medo & Culpa & Segregação & Raiva & Ajuda & Evitação & Coerção \\
\hline \multirow{4}{*}{$\begin{array}{l}\text { AP de } \\
\text { doença } \\
\text { mental }\end{array}$} & Médias & Sem AP & 5,44 & 4,04 & 4,46 & 1,43 & 4,15 & 2,98 & 6,44 & 2,14 & 5,69 \\
\hline & resposta & Com AP & 5.70 & 4,36 & 471 & 174 & 4.17 & 268 & 638 & 229 & 6,50 \\
\hline & \multirow{2}{*}{\multicolumn{2}{|c|}{$\begin{array}{l}\text { Teste estatístico } \\
p \text {-value }\end{array}$}} & TS & TS & TS & MW & TS & TS & TS & TS & TS \\
\hline & & & 0,509 & 0,375 & 0,500 & 0,328 & 0,955 & 0,385 & 0,857 & 0,588 & 0,058 \\
\hline \multirow{5}{*}{$\begin{array}{l}\text { AF de } \\
\text { doença } \\
\text { mental }\end{array}$} & Médias & Sem AF & 5,55 & 3,99 & 4,54 & 1,43 & 4,30 & 3,05 & 6,19 & 2,20 & 6,13 \\
\hline & de & & & & & & & & & & \\
\hline & resposta & Com AF & 5,52 & 4,44 & 4,59 & 1,65 & 3,92 & 2,56 & 6,79 & 2,19 & 5,79 \\
\hline & teste esta & tístico & TS & TS & TS & MW & TS & TS & TS & TS & TS \\
\hline & $p$-value & & 0,942 & 0,207 & 0,900 & 0,306 & 0,367 & 0,146 & 0,063 & 0,957 & 0,426 \\
\hline
\end{tabular}

Legenda: TS: T-Student; MW; Mann-Whitney; AP: Antecedentes Pessoais; AF: Antecedentes Familiares.

integração do doente mental na sociedade. O médico de família, enquanto médico mais próximo da comunidade, deve estar atento a esta problemática, tentando minimizar o estigma através da educação dos seus utentes, visando a melhoria dos cuidados e apoio ao doente e à família.

\section{AGRADECIMENTOS}

As autoras gostariam de agradecer a todos aqueles que de alguma forma ajudaram na aplicação deste estudo.

Gostaríamos de destacar particularmente as colegas Davina Bento, Sara Martins e Susana Machado, internas de Medicina Geral e Familiar, pela preciosa colaboração na recolha de dados. 


\section{REFERÊNCIAS BIBLIOGRÁFICAS}

1. Barbosa T. Estigma face à doença mental por parte dos futuros profissionais de saúde mental [Dissertation]. Porto: Faculdade de Psicologia e Ciências da Educação da Universidade do Porto; 2010. Portuguese

2. Corbière $M$, Samson E, Villotti P, Pelletier JF. Strategies to fight stigma toward people with mental disorders: perspectives from different stakeholders. Scientific World J. 2012;2012:516358.

3. Boyd JE, Katz EP, Link BG, Phelan JC. The relationship of multiple aspects of stigma and personal contact with someone hospitalized for mental illness, in a nationally representative sample. Soc Psychiatry Psychiatr Epidemiol. 2010;45(11):1063-70.

4. Svensson B, Markström U, Bejerholm U, Björkman T, Brunt D, Eklund $M$ et al. Test - retest reliability of two instruments for measuring public attitudes towards persons with mental illness. BMC Psychiatry. 2011;11:11.

5. Bayar MR, Poyraz PC, Aksoy-Poyraz C, Arikan MK. Reducing mental illness stigma in mental health professionals using a web-based approach. Isr J Psychiatry Relat Sci. 2009;46(3):226-30.

6. Kassam A, Papish A, Modgill G, Patten S. The development and psychometric properties of a new scale to measure mental illness related stigma by health care providers: the Opening Minds Scale for Health Care Providers (OMS-HC). BMC Psychiatry. 2012;12:62.

7. Corrigan P, Markowitz FE, Watson A, Rowan D, Kubiak MA. An attribution model of public discrimination towards persons with mental illness. J Health Soc Behav. 2003;44(2):162-79.

8. Corrigan P. A toolkit for evaluating programs meant to erase the stig- ma of mental illness. Illinois Institute of Technology; 2012.

9. Ministério da Saúde. Plano nacional de saúde 2004-2010. Lisboa: MS; 2004. Portuguese

10. Gomes J, Machado A, Cavadas LF, Teixeira H, Pires P, Santos JA, et al. Perfil do hiperfrequentador nos cuidados de saúde primários [The primary care frequent atender profile]. Acta Med Port. 2013;26(1):17-23. Portuguese

11. Corrigan PW, Morris SB, Michaels PJ, Rafacz JD, Rüsch N. Challenging the public stigma of mental illness: a meta-analysis of outcome studies. Psychiatr Serv. 2012;63(10):963-73.

12. Dyduch A, Grzywa A. [Stigma and related factors basing on mental illness stigma]. Pol Merkur Lekarski. 2009;26(153):263-7. Polish

13. Corrigan PW, Watson AC. The stigma of psychiatric disorders and the gender, ethnicity, and education of the perceiver. Community Ment Health J. 2007;43(5):439-58.

\section{CONFLITOS DE INTERESSE}

Os autores declaram não ter conflitos de interesses.

\section{ENDEREÇO PARA CORRESPONDÊNCIA}

Ana Rita Fernandes Oliveira

Rua Dr. José Oliveira Baptista, $6-2^{\circ} \mathrm{dt}^{\circ}, 2300-491$ Tomar

E-mail: rita-olive@hotmail.com

Recebido em 18-11-2013

Aceite para publicação em 19-07-2014

\section{ABSTRACT}

\section{STIGMA IN MENTAL ILLNESS}

Objectives: To estimate the prevalence of stigma in relation to mental illness in a general practice population and its association with socio-demographic characteristics or previous contact with mental illness.

Type of study: Cross-sectional

Setting: Unidade de Saúde Familiar Santiago do Centro de Saúde Arnaldo Sampaio (Leiria) and Centro de Saúde da Marinha Grande. Population: Patients 18 years of age and older registered in the two health units

Methods: A convenience sample of patients was used. Socio-demographic factors, a personal history and a family history of mental illness were assessed. The AQ-9 questionnaire of Corrigan was used to measure stigma. Univariate and multivariate analysis were performed with significance set at the 0.05 level.

Results: The study sample consisted of 206 patients with an average of 45 years. The attributes of mental illness receiving the highest scores in the AQ-9 questionnaire were the need for help, coercion for treatment, and segregation from the community. Married patients and those with children had the greatest agreement with the statement regarding coercion for treatment (Student t $p=0.008$, ANOVA $p=0.018$ ). A lower educational level was associated with higher agreement with statement regarding the need for segregation of the mentally ill (ANOVA $p<0.001$ ). No association was found between stigma and a personal or family history of mental illness.

Conclusion: Evidence of stigma from mental illness was found in this population. Marital status and education were associated with stigma. No association was found between demographic variables and other items in the AQ-9.

MeSH terms: Stigma; Mental Illness. 